10

\title{
Associations between serum levels of calcium, parathyroid hormone and future risk of venous thromboembolism: the Tromsø study
}

\author{
Gunhild Lerstad', Ellen E Brodin ${ }^{1,4}$, Johan Svartberg ${ }^{2,3}$, Rolf Jorde ${ }^{2,3}$, Jan Brox ${ }^{1,5}$, \\ Sigrid K Brækkan ${ }^{1,3}$ and John-Bjarne Hansen 1,3 \\ ${ }^{1}$ Department of Clinical Medicine, K.G. Jebsen Thrombosis Research and Expertise Center (TREC) and \\ ${ }^{2}$ Department of Clinical Medicine, Endocrine Research Group, University of Tromsø - The Arctic \\ University of Norway, Tromsø, Norway, ${ }^{3}$ Division of Internal Medicine, University Hospital of North \\ Norway, Tromsø, Norway, ${ }^{4}$ Division of Internal Medicine, Akershus University Hospital, Lørenskog, \\ Norway, and ${ }^{5}$ Department of Laboratory Medicine, University Hospital of North Norway, Tromsø, Norway
}

\author{
Correspondence \\ should be addressed \\ to $\mathrm{G}$ Lerstad \\ Email \\ gunhild.lerstad@uit.no
}

\begin{abstract}
Objective: The relationship between serum levels of calcium, parathyroid hormone (PTH) and risk of venous thromboembolism (VTE) has not been addressed in population-based cohorts. We investigated the associations between serum levels of calcium and PTH, with future risk of VTE in a general adult population.

Design: Population-based cohort.

Methods: A total of 27712 subjects (25-87 years) who participated in Tromsø 4 (1994-1995) and Tromsø 5 (2001-2002) surveys were included in the study, and total calcium and PTH were measured in 27685 and 8547 subjects respectively. Incident VTE was recorded through December 31, 2012. Cox-regression models with calcium and PTH as time-varying exposures were used to calculate hazard ratios (HR) of VTE by quartiles of calcium and PTH. Quartiles of calcium and PTH were also combined to assess the effect of discordants of both PTH and calcium (e.g. highest and lowest quartiles of both calcium and PTH) on VTE risk using the middle two quartiles as reference.

Results: There were 712 VTEs during 15.0 years of median follow-up. Serum levels of calcium and PTH were not associated with risk of VTE. However, subjects with discordant high serum levels of both calcium and PTH (calcium $\geq 2.45 \mathrm{mmol} / \mathrm{L}$ and PTH $\geq 4.0 \mathrm{pmol} / \mathrm{L}$ ) had increased risk of VTE compared to those in subjects with normal calcium and PTH (multivariable HR: $1.78,95 \% \mathrm{Cl}: 1.12-2.84)$.

Conclusions: Serum levels of calcium and PTH separately were not associated with future risk of VTE, but subjects with high levels of both calcium and PTH had increased risk of VTE compared to those in subjects with normal levels.
\end{abstract}

\section{Introduction}

Parathyroid dysfunction is among the most common endocrine disorders. Primary hyperparathyroidism, with an annual incidence of about 20 cases per 100 000, is the most frequent (1), caused by autonomous production of parathyroid hormone (PTH) by one or more of the parathyroid glands. PTH is a key hormone in calcium
() 2017 European Society of Endocrinology Printed in Great Britain homeostasis with an inverse relation to ionized calcium under normal conditions. Low serum ionized calcium triggers the secretion of PTH from the parathyroid glands resulting in a rise in serum ionized calcium, whereas high serum levels of ionized calcium inhibits PTH secretion (2). Patients with primary hyperparathyroidism,

Published by Bioscientifica Ltd. 
and consequently, chronic hypercalcemia have increased plasma levels of hemostatic factors and coagulation activators, including factor VII (FVII) and X (FX), D-dimer and plasminogen activator inhibitor-1 (PAI-1), which may induce a hypercoagulable state in these patients $(3,4,5)$. Population-based cohort studies have also demonstrated that serum calcium and PTH are independent risk factors for myocardial infarction in middle-aged men (6) and cardiovascular mortality in elderly men (7) respectively.

Venous thromboembolism (VTE), comprising deep vein thrombosis (DVT) and pulmonary embolism (PE), is a common disorder with serious short-term and longterm complications, and a potentially fatal outcome $(8$, 9). The incidence of VTE is 1-2 per 1000 persons per year in the general population, with a steep incline with age. Even though many environmental and inherited risk factors have been associated with VTE $(8,9,10,11,12)$, still many of the events occur without any obvious risk factor $(13,14,15)$. Therefore, it is important to identify biomarkers and risk behaviors of VTE that could be subject to modification to reduce the disease burden.

Risk factors for VTE often involve an imbalance of the hemostatic system in a prothrombotic direction $(16,17)$. Therefore, a possible hypercoagulable state in patients with primary hyperparathyroidism could potentially imply an increased risk of VTE in these patients. To the best of our knowledge, the relationship between serum calcium, PTH and the future risk of VTE has not previously been addressed. We therefore set out to examine the associations between serum levels of calcium and PTH and future risk of VTE in a general adult population.

\section{Subjects and methods}

\section{Study population}

Participants were recruited from the fourth and fifth surveys of the Tromsø study (conducted in 1994-1995 and 2001-2002 respectively) (18). To the fourth survey, all men and women aged $\geq 25$ years living in the municipality of Tromsø, Norway, were invited to participate. A subgroup was invited to a second more extensive screening that included blood samples for hormone analysis. In the fifth survey, all men and women, who participated in the second screening of the fourth survey or became 30 , $40,45,60$ or 75 years old during 2001, were invited to participate. The overall attendance rate was close to $80 \%$ in both surveys. A total of 27844 unique individuals aged 25-87 years participated in at least one survey and were thereby eligible for our study. A detailed description of

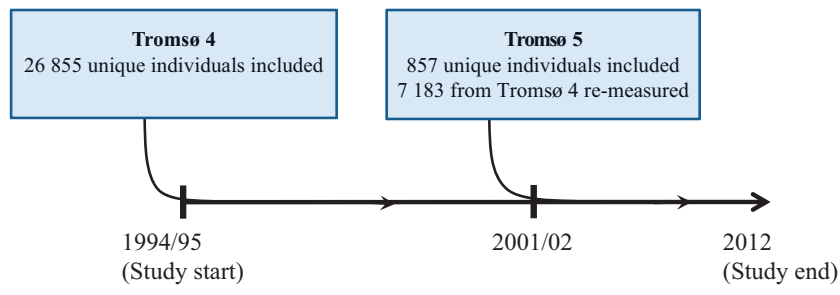

Figure 1

Flow chart of the follow-up of subjects included from the fourth (1994/1995) and fifth (2001/2002) survey of the Tromsø study.

study participation has been published elsewhere (18). The study was approved by the Regional Committee of Medical and Health Research Ethics, and all participants gave their informed written consent to participate. Subjects who were not officially registered as inhabitants of the municipality of Tromsø at baseline $(n=22)$, and subjects with a known pre-baseline history of VTE $(n=59)$ were excluded from the study. Furthermore, subjects were excluded if they had missing values of calcium and PTH $(n=51)$ in both visits. In total, 27712 subjects were included in the study (Fig. 1) and followed from the date of enrollment through the end of the study period, December 31, 2012.

\section{Measurements}

Baseline information was collected by physical examinations, blood samples and self-administered questionnaires (19). Information on arterial cardiovascular diseases (CVD) (angina pectoris, myocardial infarction (MI) and stroke), current daily smoking and physical activity ( $\geq 1 \mathrm{~h}$ per week) during leisure time was collected from the questionnaires. Height and weight were measured, and body mass index (BMI) was calculated as weight in kilograms divided by the square of height in meters $\left(\mathrm{kg} / \mathrm{m}^{2}\right)$. Blood pressure was recorded with an automatic device (Dinamap Vital Signs Monitor 1846; Critikon Inc., Tampa, FL, USA). Participants rested for $2 \mathrm{~min}$ in a sitting position before three readings were taken on the upper right arm at 2-min intervals. The average of the two last readings was used in the analysis. Non-fasting blood samples were collected from an antecubital vein, serum prepared by centrifugation after $1 \mathrm{~h}$ respite at room temperature, and further analyzed at the Department of Clinical Chemistry, University Hospital of North Norway. The Hitachi Model 917 analyzer was used to quantify total serum concentrations of calcium and creatinine with 
reagents from Boehringer Mannheim (Mannheim, FRG). The reference range for calcium was $2.15-2.51 \mathrm{mmol} / \mathrm{L}$. The reference range for creatinine in men was $60-105 \mu \mathrm{mol} / \mathrm{L}$ and $45-90 \mu \mathrm{mol} / \mathrm{L}$ for women. Creatinine values were used for the estimation of the glomerular filtration rate (eGFR). eGFR was calculated using the recalibrated four-variable Modification of Diet in Renal Disease $(\mathrm{MDRD})$ study equation; $\mathrm{eGFR}=175 \times$ (s-creatinine $(\mu \mathrm{mol} / \mathrm{L}) / 88.4)^{-1.154} \times$ age $^{-0.203} \times(0.742$ if female) $(20)$ Intact PTH was measured by an IMMULITE analyzer (Diagnostic Products, Los Angeles, CA, USA) on the basis of a two-site chemiluminescent immunometric assay. The reference range was $1.1-6.8 \mathrm{pmol} / \mathrm{L}$ for those below the age of 50 years, and $1.1-7.5 \mathrm{pmol} / \mathrm{L}$ for those aged 50 years and above. In the fourth Tromsø study, serum calcium was measured within a week after sampling, whereas serum PTH was measured in 2001. In the fifth Tromsø study, calcium and PTH were measured within 2 and 12 months respectively. All samples were stored frozen at $-70^{\circ} \mathrm{C}$. The Cobas Mira instrument was used to quantify $\mathrm{HbA1c}$ with an immunoturbidimetric method (Unimate $5 \mathrm{HbA1c}$, Hoffmann-La Roche). The reference range was 4.0-6.5\%. Serum total cholesterol and triglycerides were analyzed by enzymatic colorimetric methods and commercially available kits (CHOD-PAP for cholesterol and GPO-PAP for triglycerides; Boehringer-Mannheim, Mannheim, Germany). Serum HDL cholesterol was measured after precipitation of lower-density lipoproteins with heparin and manganese chloride.

\section{Venous thromboembolism ascertainment}

All incident VTE events during follow-up were identified by searching the discharge diagnosis registry, the autopsy registry and the radiology procedure registry at the University Hospital of North Norway as previously described (21). The University Hospital of North Norway is the only hospital in the region, and all diagnostic radiology and hospital care is provided exclusively by this hospital. The medical record for each potential case of VTE was reviewed by trained personnel, and a VTE event was considered verified and recorded when the presence of clinical signs and symptoms of DVT or pulmonary embolism were combined with objective confirmation tests (by compression ultrasonography, venography, spiral computed tomography, perfusion-ventilation scan, pulmonary angiography and autopsy) and resulted in a VTE diagnosis that required treatment. VTE cases from the autopsy registry were recorded when the death certificate indicated VTE as a cause of death or a significant condition associated with death. Concurrent DVT and PE were registered as $\mathrm{PE}$, and only the first lifetime symptomatic, objectively confirmed VTE events during follow-up were included in the analyses. Among patients diagnosed with DVT, only patients with clinical signs and symptoms that indicated PE were screened for PE. Based on the presence of provoking factors at the time of diagnosis, the VTE event was classified as unprovoked (no provoking factors) or provoked ( $\geq$ one provoking factors). Major surgery, trauma or an acute medical condition (acute MI, ischemic stroke or major infectious disease) within 8 weeks prior to the event, active cancer at the time of the event or marked immobilization (e.g. bed rest $\geq 3$ days, wheelchair, or long distance travels $\geq 4 \mathrm{~h}$ within 14 days prior to the event) were considered as provoking factors.

\section{Statistical analysis}

Date of the study enrollment for each individual was determined as the date of attendance in the first survey in which calcium or PTH measurements was available. Person-years were accrued from the date of enrollment to the date a VTE event was first diagnosed, the date of migration or death or at the end of the study period (December 31, 2012), whichever came first. We used a time-varying analysis that allowed participants $(n=7183)$ who were re-measured in Tromsø 5 to change (update) levels of PTH and calcium over time. In total, 27712 individuals contributed with 34605 observational periods.

Statistical analysis was carried out using STATA version 14.0 (Stata Corporation, College Station, TX, USA). Cox-proportional hazards regression models were used to estimate hazard ratios (HR) with 95\% confidence intervals (CI) for all VTE events, as well as for DVT and $\mathrm{PE}$, and for provoked and unprovoked VTE, by increasing levels of calcium and PTH. Calcium, PTH and potential confounders were entered as time-varying co-variates. Age was used as time-scale, and the subjects' age at study enrollment was defined as entry-time, and exit-time was defined as age at date of VTE diagnosis, death, migration or study end. HRs for all VTE events were estimated in analyses adjusted for age (time-scale) and sex, and in multivariable analyses with further adjustment for BMI. Multivariable adjusted HRs of VTE according to continuous levels of calcium and PTH were visualized by generalized additive regression plots. In these plots, the exposures (log transformed calcium and PTH respectively) were modeled with a 4-degree of freedom smoothing spline fit in 
Cox-proportional hazard models including the same co-variates as described above. HRs of VTE according to quartiles of calcium ( $<25$ th percentile: $1.77-2.31 \mathrm{mmol} / \mathrm{L}$, 25-50th percentile: $2.32-2.37 \mathrm{mmol} / \mathrm{L}, 50-75$ th percentile: $2.38-2.44 \mathrm{mmol} / \mathrm{L},>75$ th percentile: $2.45-3.00 \mathrm{mmol} / \mathrm{L}$ ) and to quartiles of PTH $(<25$ th percentile: $0.1-2.1 \mathrm{pmol} / \mathrm{L}$, 25-50th percentile: $2.2-2.9 \mathrm{pmol} / \mathrm{L}, 50-75$ th percentile: $3.0-3.9 \mathrm{pmol} / \mathrm{L},>75$ th percentile: $4.0-26.8 \mathrm{pmol} / \mathrm{L}$ ) were calculated and the first quartile in both models was used as the reference category. Cox-regression models were additionally performed to test for linear trends across these categories.

By combining the quartiles of calcium with the quartiles of PTH, wherein quartile 2 and 3 of both calcium and PTH was first merged to one category (2575th percentile), totally nine combined categories of PTH and calcium were generated, making it possible to assess the effect of the extremities and discordants of PTH- and calcium levels on VTE risk. These categories were defined as 'low' ( $<25$ th percentile), 'normal' (25-75th percentile) and 'high' ( $<75$ th percentile). The category comprising 'normal' calcium and PTH levels only (calcium: 2.32$2.44 \mathrm{mmol} / \mathrm{L}$ and PTH: $2.2-3.9 \mathrm{pmol} / \mathrm{L}$ ) was used as the reference category. We also examined the apparent effects of discordant levels of PTH and calcium on VTE risk in a cause-specific analysis where subjects who developed MI, stroke or cancer were censored at the date of event. Events of MI, stroke and cancer were validated by an independently endpoint-committee as previously described $(22,23,24)$.
Statistical interactions with sex were tested for all risk factors by including cross-product terms in the proportional hazard models, and no interactions were found. The proportional hazard assumption was verified by the use of Schoenfeld residuals and by evaluating the parallelism between the curves of the log-log survivor function for different categories of calcium and PTH.

\section{Results}

Baseline characteristics of study participants across quartiles of total serum calcium are shown in Table 1. Subjects with calcium in the upper quartile $(2.45-3.00 \mathrm{mmol} / \mathrm{L})$ had higher BMI, blood pressure, triglycerides, total cholesterol and HbA1c. Furthermore, subjects in the upper quartile were more likely smokers, less physical active and they were less frequently women compared to those in the lowest quartile. As expected, we observed an inverse relationship between calcium and PTH (Table 1). Baseline characteristics of participants across quartiles of serum PTH are shown in the Supplementary Table 1 (see section on supplementary data given at the end of this article). Subjects with PTH in the upper quartile $(4.0-26.8 \mathrm{pmol} / \mathrm{L})$ were older and had higher BMI, blood pressure and triglycerides, lower HDL cholesterol and were less physically active. Subjects in the upper quartile of PTH were also less likely smokers and had a higher proportion of prior CVD (Supplementary Table 1).

Table 1 Baseline characteristics across quartiles of total serum calcium concentrations of subjects enrolled in the Tromsø study (94/95 and 01/02). Values are percentage with numbers in brackets or means \pm S.D.

\begin{tabular}{l}
\hline \\
\hline Observation periods $(n)$ \\
Age (years) \\
Sex $(\%$ women) \\
BMI $\left(\mathrm{kg} / \mathrm{m}^{2}\right)$ \\
SBP $(\mathrm{mmHg})$ \\
DBP $(\mathrm{mmHg})$ \\
Triglycerides $(\mathrm{mmol} / \mathrm{L})^{\dagger}$ \\
Total cholesterol $(\mathrm{mmol} / \mathrm{L})$ \\
HDL-cholesterol $(\mathrm{mmol} / \mathrm{L})$ \\
Creatinine $(\mu \mathrm{mol} / \mathrm{L})$ \\
eGFR (mL/min/1.73 $\left.\mathrm{m}^{2}\right)^{\ddagger}$ \\
HbA1c $(\%)$ \\
PTH (pmol/L) \\
Smoking $(\%)$ \\
Physical activity $(\%)^{\S}$ \\
CVD $(\%) *$
\end{tabular}

\begin{tabular}{c}
\hline $\mathbf{1 . 7 7 - 2 . 3 1}$ \\
\hline $27(9352)$ \\
$49 \pm 16$ \\
$58.3(5455)$ \\
$25.2 \pm 4.00$ \\
$132 \pm 20$ \\
$76 \pm 12$ \\
$1.18(0.82-1.74)$ \\
$5.74 \pm 1.21$ \\
$1.48 \pm 0.40$ \\
$84 \pm 19$ \\
$91 \pm 19$ \\
$5.4 \pm 0.7$ \\
$3.52 \pm 1.79$ \\
$32.9(3069)$ \\
$31.5(2800)$ \\
$8.1(755)$
\end{tabular}

\begin{tabular}{c}
\hline $\mathbf{2 . 3 2 - 2 . 3 7}$ \\
\hline $24(8121)$ \\
$50 \pm 16$ \\
$53.6(4348)$ \\
$25.5 \pm 3.94$ \\
$135 \pm 20$ \\
$78 \pm 12$ \\
$1.25(0.88-1.86)$ \\
$6.01 \pm 1.25$ \\
$1.48 \pm 0.40$ \\
$84 \pm 18$ \\
$91 \pm 20$ \\
$5.5 \pm 0.8$ \\
$3.23 \pm 1.53$ \\
$34.9(2826)$ \\
$32.6(2488)$ \\
$8.4(674)$
\end{tabular}

\begin{tabular}{c}
$\mathbf{2 . 3 8 - 2 . 4 4}$ \\
\hline $26(9137)$ \\
$50 \pm 15$ \\
$51.2(4683)$ \\
$25.5 \pm 3.9$ \\
$137 \pm 21$ \\
$79 \pm 12$
\end{tabular}

$\mathbf{2 . 4 5}-\mathbf{3 . 0 0}$
$23(7888)$
$49 \pm 16$
$50.2(3957)$
$25.8 \pm 4.0$
$140 \pm 21$
$81 \pm 13$

$1.31(0.91-1.96)$

$6.17 \pm 1.27$

$1.50 \pm 0.41$

$83 \pm 17$

$91 \pm 18$

$5.5 \pm 0.8$

$3.02 \pm 1.45$

$35.5(3238)$

$31.8(2762)$

$1.47(0.98-2.10)$

$6.43 \pm 1.32$

$1.51 \pm 0.41$

$81 \pm 21$

$90 \pm 18$

$5.5 \pm 0.7$

$3.08 \pm 1.82$

36.7 (2895)

29.2 (2211)

8.3 (655)

*self-reported history of myocardial infarction, angina pectoris or stroke; ${ }^{\top}$ Values are median with interquartile range in brackets; ${ }^{\ddagger}$ only available in 15301 of total 34498 observation periods (44\%); ${ }^{\aleph_{s}}$ weat production and breathlessness $\geq 1 \mathrm{~h}$ per week during leisure time. 
Table 2 Characteristics of VTE patients $(n=712)$ at the time of the VTE diagnosis. The Tromsø study.

\begin{tabular}{|c|c|}
\hline & $\%(n)$ \\
\hline Deep vein thrombosis & $58.1(414)$ \\
\hline Pulmonary embolism & $41.9(298)$ \\
\hline Unprovoked** & $41.6(296)$ \\
\hline \multicolumn{2}{|l|}{ Clinical risk factors } \\
\hline Estrogens* & $5.0(48)$ \\
\hline Heredity $^{\dagger}$ & $2.7(26)$ \\
\hline Pregnancy & $0.1(1)$ \\
\hline Other medical conditions ${ }^{\ddagger}$ & $22.6(216)$ \\
\hline \multicolumn{2}{|l|}{ Provoking factors } \\
\hline Surgery & $15.0(144)$ \\
\hline Trauma & $8.4(80)$ \\
\hline Acute medical conditions & $14.6(140)$ \\
\hline Cancer & $24.1(231)$ \\
\hline Immobilization (bed rest $>3$ days, wheelchair) & $23.3(176)$ \\
\hline Other $§$ & $5.3(51)$ \\
\hline
\end{tabular}

**No provoking factors at the time of diagnosis; *Hormone replacement therapy/oral contraceptives; theredity: family history of VTE in firstdegree relative before the age of 60 years; ${ }^{\ddagger}$ other diseases within the previous year (myocardial infarction, ischemic stroke, heart failure, inflammatory bowel disease, chronic infections, chronic obstructive pulmonary disease or myeloproliferative disorders); ${ }^{\S}$ other factor specifically described as provoking in the medical record (e.g. intravascular catheter).

VTE, venous thromboembolism.

There were 712 validated incident VTE events during a total of 100696 person-years of follow-up. The median time from calcium and/or PTH measurement to the end of each follow-up period was 11.6 years (range: 0.02-18.32), whereas the overall median follow-up time was 15.0 years (range: 0.02-18.32). The overall crude incidence rate of VTE was 1.77 per 1000 person-years (95\% CI: 1.64-1.90). Characteristics of VTE patients at the time of the event are shown in Table 2. Among the subjects with incident VTE, $58.1 \%$ had DVT, $41.9 \%$ had PE, and 296 events (41.6\%) were classified as unprovoked. Cancer was the most common provoking factor $(24.1 \%$ of the VTE patients had a cancer-related VTE), followed by immobilization (23.3\%) (Table 2).

When analyzing calcium as a continuous variable, no association was found between levels of calcium and VTE after adjustments for potential confounders (Fig. 2). The incidence rates and relative risks (HRs) of total VTE and subtypes of VTE across quartiles of total serum calcium are shown in Table 3. Subjects in the highest quartile of serum calcium did not have higher risk of total VTE or subtypes of VTE compared to subjects in the lowest quartile of VTE in either age- and sex adjusted analyses or multivariable analyses. Furthermore, there was no linear trend in increased risk of total VTE or subtypes

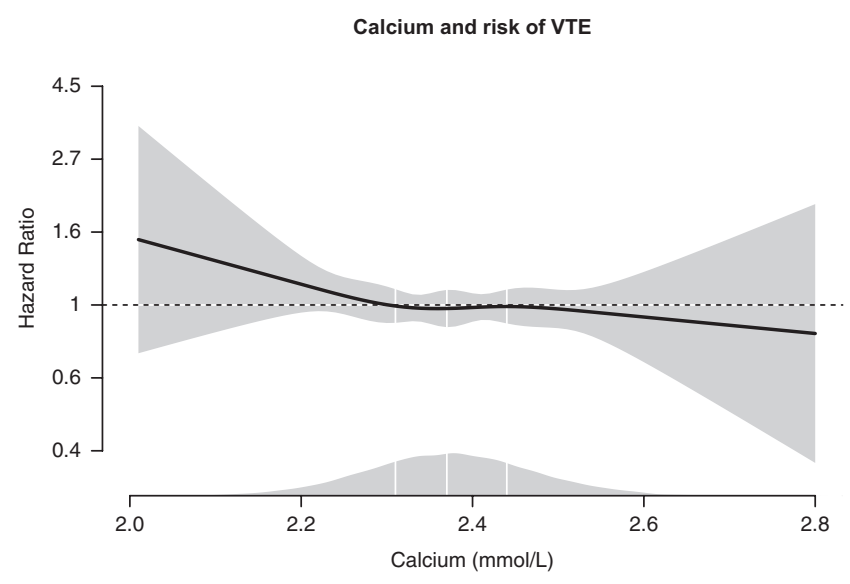

Figure 2

Dose-response relationship between calcium and risk of venous thromboembolism (VTE) obtained by generalized additive regression. The regression model is adjusted for age, sex and body mass index. The solid line shows hazard ratios and the shaded area shows $95 \%$ confidence intervals. Density plots show the distribution of calcium, and white vertical lines indicate 2.5 th, 25th, 50th, 75th and 97.5th percentiles.

of VTE across categories of increasing total serum calcium. Similar analyses were performed to explore the association between serum PTH and future risk of total VTE and subtypes of VTE (Table 4). No association was found between categories of serum PTH and future risk of total VTE or subtypes of VTE. Moreover, there was no association between levels of PTH and VTE when PTH was analyzed as a continuous variable (Fig. 3).

In order to investigate whether discordants of the PTH-calcium axis (e.g. highest and lowest quartiles of both PTH and calcium respectively) would influence the VTE risk, we combined quartiles of serum calcium and PTH using the middle two quartiles (25-75th percentile) as reference (Table 5). The subjects within the highest categories of calcium and PTH (calcium $\geq 2.45 \mathrm{mmol} / \mathrm{L}$ and PTH $\geq 4.0 \mathrm{pmol} / \mathrm{L}$ ) had 1.8-fold higher risk (HR 1.75, 95\% CI: 1.10-2.78) of VTE compared with those with serum calcium and PTH in the two middle quartiles. In the multivariable model, the risk estimate increased slightly (multivariable HR: 1.78, 95\% CI: 1.12-2.84 (Table 5). Further adjustments for smoking, HbA1c and eGFR did not alter the results (data not shown). The risk estimates for VTE by the highest categories of calcium and PTH remained essentially unchanged after censoring for MI and stroke (multivariable HR: 1.63, 95\% CI: 0.951.60), and when censoring for cancer (multivariable HR: 1.76, 95\% CI: 1.01-3.09) in the cause-specific analysis. 
Table 3 Baseline characteristics across quartiles of total serum calcium concentrations of subjects enrolled in the Tromsø study (94/95 and 01/02) incidence rates (IR) and hazard ratios (HR) of total venous thromboembolism (VTE) and VTE subtypes across quartiles of total serum calcium concentrations.

\begin{tabular}{|c|c|c|c|c|c|}
\hline & \multicolumn{4}{|c|}{ Calcium (mmol/L) } & \multirow[b]{2}{*}{$P$ for trend } \\
\hline & $1.77-2.31$ & $2.32-2.37$ & $2.38-2.44$ & $2.45-3.00$ & \\
\hline \multicolumn{6}{|l|}{ Total VTE } \\
\hline Person-years & 108483 & 94571 & 105975 & 92788 & \\
\hline Events & 198 & 171 & 179 & 160 & \\
\hline$I^{*}$ & $1.83(1.59-2.10)$ & $1.81(1.56-2.10)$ & 1.69 (1.46-1.96) & $1.72(1.48-2.01)$ & \\
\hline $\mathrm{HR}^{\dagger}$ & 1.00 (ref.) & $0.95(0.78-1.17)$ & $0.93(0.76-1.13)$ & $0.97(0.79-1.20)$ & 0.71 \\
\hline $\mathrm{HR}^{\ddagger}$ & 1.00 (ref.) & $0.94(0.76-1.15)$ & $0.89(0.73-1.09)$ & $0.95(0.77-1.17)$ & 0.48 \\
\hline \multicolumn{6}{|l|}{ DVT } \\
\hline Person-years & 107751 & 93947 & 105302 & 92263 & \\
\hline Events & 111 & 99 & 102 & 100 & \\
\hline$I^{*}$ & $1.03(0.85-1.24)$ & $1.05(0.87-1.28)$ & $0.97(0.80-1.18)$ & $1.08(0.89-1.32)$ & \\
\hline $\mathrm{HR}^{\dagger}$ & 1.00 (ref.) & $0.99(0.75-1.29)$ & $0.94(0.72-1.23)$ & $1.07(0.82-1.41)$ & 0.75 \\
\hline $\mathrm{HR}^{\ddagger}$ & 1.00 (ref.) & $0.96(0.73-1.26)$ & $0.88(0.67-1.16)$ & $1.04(0.79-1.36)$ & 0.98 \\
\hline \multicolumn{6}{|l|}{$\mathrm{PE}$} \\
\hline Person-years & 107770 & 93845 & 105252 & 92113 & \\
\hline Events & 87 & 72 & 77 & 60 & \\
\hline$I^{*}$ & $0.81(0.65-1.00)$ & $0.77(0.61-0.97)$ & $0.73(0.59-0.91)$ & $0.65(0.51-0.84)$ & \\
\hline $\mathrm{HR}^{+}$ & 1.00 (ref.) & $0.92(0.67-1.25)$ & $0.92(0.67-1.25)$ & $0.85(0.61-1.18)$ & 0.35 \\
\hline $\mathrm{HR}^{\ddagger}$ & 1.00 (ref.) & $0.91(0.67-1.24)$ & $0.90(0.66-1.23)$ & $0.83(0.59-1.15)$ & 0.28 \\
\hline \multicolumn{6}{|l|}{ Provoked VTE } \\
\hline Person-years & 107889 & 93979 & 105398 & 92274 & \\
\hline Events & 119 & 97 & 108 & 91 & \\
\hline$I^{*}$ & $1.10(0.92-1.32)$ & $1.03(0.85-1.26)$ & $1.02(0.85-1.24)$ & $0.99(0.80-1.21)$ & \\
\hline $\mathrm{HR}^{\dagger}$ & 1.00 (ref.) & $0.90(0.69-1.18)$ & $0.93(0.72-1.21)$ & $0.93(0.71-1.22)$ & 0.65 \\
\hline $\mathrm{HR}^{\ddagger}$ & 1.00 (ref.) & $0.88(0.67-1.15)$ & $0.90(0.69-1.17)$ & $0.91(0.69-1.19)$ & 0.49 \\
\hline \multicolumn{6}{|l|}{ Unprovoked VTE } \\
\hline Person-years & 107633 & 93814 & 105155 & 92102 & \\
\hline Events & 79 & 74 & 71 & 69 & \\
\hline$I R^{*}$ & $0.73(0.59-0.92)$ & $0.79(0.63-0.99)$ & $0.68(0.54-0.85)$ & $0.75(0.59-0.95)$ & \\
\hline $\mathrm{HR}^{\dagger}$ & 1.00 (ref.) & $1.04(0.76-1.43)$ & $0.92(0.67-1.27)$ & $1.04(0.75-1.44)$ & 0.99 \\
\hline $\mathrm{HR}^{\ddagger}$ & 1.00 (ref.) & $1.03(0.75-1.42)$ & $0.88(0.64-1.22)$ & $1.01(0.73-1.40)$ & 0.80 \\
\hline
\end{tabular}

*Incidence rate per 1000 person-years; ${ }^{\dagger}$ adjusted for age as time-scale and sex; ${ }^{\ddagger}$ adjusted for age as time-scale, sex and body mass index.

Likewise, subjects within the lowest categories of calcium and PTH (calcium $<2.32 \mathrm{mmol} / \mathrm{L}$ and PTH $<2.1 \mathrm{pmol} / \mathrm{L}$ ) had 1.6-fold higher risk (multivariable HR: 1.63, 95\% CI: 0.98-2.69) of VTE compared with those with serum calcium and PTH in the two middle quartiles (Table 5). In cause-specific analysis where the impact of cancer was eliminated, the association between the lowest categories of calcium and PTH and VTE risk was strengthened (multivariable HR: 1.81, 95\% CI: 1.02-3.21).

\section{Discussion}

In the present study, we aimed to investigate whether serum levels of calcium and PTH influenced the risk of VTE. We confirmed an inverse association between serum levels of PTH and calcium, and found that neither increased serum levels of PTH nor total serum calcium alone were associated with future risk of total VTE or
VTE subtypes. However, discordant high serum levels of both PTH and total calcium were associated with a $78 \%$ increased risk of VTE in multivariable analysis adjusted for age, sex and BMI.

Our results are unchallenged as this is the first population-based study to investigate the associations between serum levels of calcium and PTH, and future risk of VTE in a general population. We found that neither serum calcium nor serum PTH was associated with future risk of VTE. Our findings are supported by a randomized clinical trial reporting no effect on VTE risk by daily supplementation with calcium and vitamin D for 7 years in postmenopausal women (25). In contrast, recent metaanalyses of prospective studies have reported that both serum PTH and calcium were significantly associated with risk of arterial cardiovascular diseases and mortality $(25,26)$. We found, however, that high serum levels of both PTH and calcium was associated with increased 
Table 4 Incidence rates (IR) and hazard ratios (HR) of total venous thromboembolism (VTE) and VTE subtypes across quartiles of serum parathyroid hormone (PTH) concentrations.

\begin{tabular}{|c|c|c|c|c|c|}
\hline & \multicolumn{4}{|c|}{ PTH (pmol/L) } & \multirow[b]{2}{*}{$P$ for trend } \\
\hline & $0.1-2.1$ & $2.2-2.9$ & $3.0-3.9$ & $4.0-26.8$ & \\
\hline \multicolumn{6}{|l|}{ Total VTE } \\
\hline Person-years & 25108 & 25364 & 25517 & 25697 & \\
\hline Events & 64 & 80 & 75 & 99 & \\
\hline $\mathrm{IR}^{*}$ & $2.55(2.00-3.26)$ & $3.15(2.53-3.93)$ & $2.94(2.34-3.69)$ & $3.85(3.16-4.69)$ & \\
\hline $\mathrm{HR}^{\dagger}$ & 1.00 (ref.) & $1.08(0.78-1.50)$ & $0.89(0.64-1.25)$ & $1.09(0.79-1.50)$ & 0.84 \\
\hline $\mathrm{HR}^{\ddagger}$ & 1.00 (ref.) & $1.04(0.75-1.45)$ & $0.84(0.60-1.18)$ & $1.03(0.74-1.42)$ & 0.87 \\
\hline \multicolumn{6}{|l|}{ DVT } \\
\hline Person-years & 24952 & 25055 & 25290 & 25440 & \\
\hline Events & 40 & 36 & 42 & 55 & \\
\hline$I R^{*}$ & $1.60(1.18-2.19)$ & $1.44(1.04-1.99)$ & $1.66(1.23-2.25)$ & $2.16(1.66-2.82)$ & \\
\hline $\mathrm{HR}^{\dagger}$ & 1.00 (ref.) & $0.80(0.51-1.26)$ & $0.84(0.54-1.30)$ & $1.03(0.69-1.56)$ & 0.73 \\
\hline $\mathrm{HR}^{\ddagger}$ & 1.00 (ref.) & $0.76(0.48-1.20)$ & $0.82(0.53-1.26)$ & $1.00(0.66-1.52)$ & 0.82 \\
\hline \multicolumn{6}{|l|}{ PE } \\
\hline Person-years & 24868 & 25179 & 25287 & 25409 & \\
\hline Events & 24 & 44 & 33 & 44 & \\
\hline $\mathrm{IR}^{*}$ & $0.97(0.65-1.44)$ & $1.75(1.30-2.35)$ & $1.30(0.93-1.84)$ & $1.73(1.29-2.32)$ & \\
\hline $\mathrm{HR}^{\dagger}$ & 1.00 (ref.) & $1.52(0.93-2.51)$ & $0.97(0.57-1.65)$ & $1.19(0.72-1.96)$ & 0.93 \\
\hline $\mathrm{HR}^{\ddagger}$ & 1.00 (ref.) & $1.49(0.91-2.45)$ & $0.90(0.53-1.53)$ & $1.09(0.65-1.80)$ & 0.61 \\
\hline \multicolumn{6}{|l|}{ Provoked VTE } \\
\hline Person-years & 24925 & 25158 & 25340 & 25466 & \\
\hline Events & 35 & 50 & 49 & 59 & \\
\hline $\mathrm{IR}^{*}$ & $1.40(1.01-1.96)$ & $1.99(1.51-2.62)$ & $1.93(1.46-2.56)$ & $2.32(1.80-2.99)$ & \\
\hline $\mathrm{HR}^{\dagger}$ & 1.00 (ref.) & $1.23(0.80-1.90)$ & $1.06(0.68-1.63)$ & $1.18(0.77-1.80)$ & 0.65 \\
\hline $\mathrm{HR}^{\ddagger}$ & 1.00 (ref.) & $1.22(0.79-1.88)$ & $1.01(0.65-1.56)$ & $1.11(0.72-1.70)$ & 0.91 \\
\hline \multicolumn{6}{|l|}{ Unprovoked VTE } \\
\hline Person-years & 24895 & 25076 & 25237 & 25384 & \\
\hline Events & 29 & 30 & 26 & 40 & \\
\hline$I R^{*}$ & $1.16(0.81-1.68)$ & $1.20(0.84-1.71)$ & $1.03(0.70-1.51)$ & $1.58(1.16-2.15)$ & \\
\hline $\mathrm{HR}^{\dagger}$ & 1.00 (ref.) & $0.90(0.54-1.49)$ & $0.69(0.40-1.17)$ & $0.98(0.60-1.59)$ & 0.80 \\
\hline $\mathrm{HR}^{\ddagger}$ & 1.00 (ref.) & $0.83(0.49-1.39)$ & $0.64(0.37-1.10)$ & $0.93(0.57-1.51)$ & 0.69 \\
\hline
\end{tabular}

*Incidence rate per 1000 person-years; ${ }^{\dagger}$ adjusted for age as time-scale and sex; ${ }^{\ddagger}$ adjusted for age as time-scale, sex and body mass index.

VTE risk after adjustment for age, sex and BMI. It is not known whether the effect is mediated by indirect (e.g. arterial cardiovascular diseases) or direct prothrombotic mechanisms (e.g. prothrombotic imbalance of the hemostatic system).

In agreement with previous studies $(27,28)$, we found that high levels of serum PTH and calcium was associated with an unfavorable profile of some atherosclerotic risk factors such as high blood pressure, and high total cholesterol and triglycerides. A recent systematic review and meta-analysis of prospective studies showed a HR of 1.08 (95\% CI: 1.04-1.13) for arterial cardiovascular events by one standard deviation increase in serum calcium, which was marginally weakened by adjustment for atherosclerotic risk factors (27). Similarly, a systematic review and meta-analysis of prospective studies demonstrated that subjects in the highest quartile of serum PTH had $45 \%$ increased risk of cardiovascular events compared to those with PTH levels in the lowest quartile (26).
Among traditional atherosclerotic risk factors, only age, obesity and a family history of MI are shared risk factors for VTE $(29,30,31,32)$, whereas high blood pressure and dyslipidemia are only associated with high serum PTH and calcium $(28,33,34)$. Thus, it is unlikely that atherosclerotic risk factors could explain the association between high levels of serum PTH and calcium and VTE risk. Observational studies have displayed a transient association between MI and stroke and future risk of VTE in the general population $(23,35,36)$. Further, high serum levels of PTH and calcium are individually associated with arterial cardiovascular events (e.g. stroke and MI). Therefore, the apparent VTE risk by high levels of calcium and PTH could be mediated by arterial CVD. Additionally, hypercalcemia is relatively common in patients with cancer (37). In order to explore whether the apparent association was explained by arterial CVD (MI and stroke) and cancer, we performed cause-specific analyses where subjects were censored at the first occurring event. These findings suggest that intermediate development of arterial 
PTH and risk of VTE

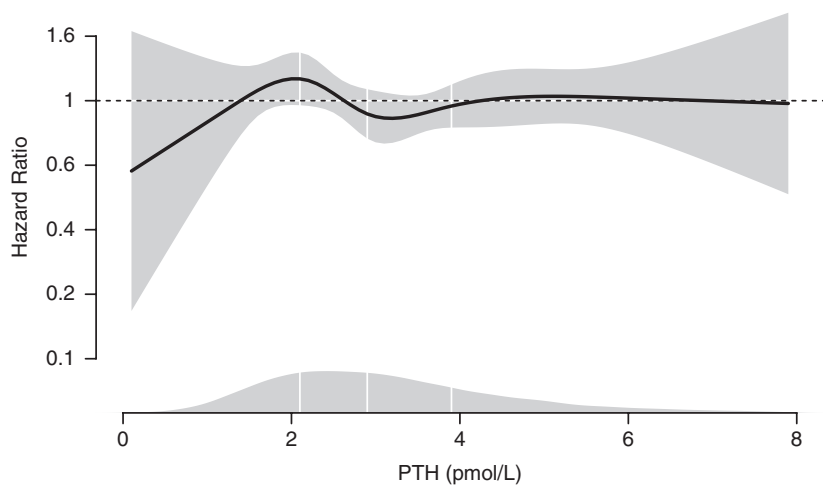

Figure 3

Dose-response relationship between parathyroid hormone (PTH) and risk of venous thromboembolism (VTE) obtained by generalized additive regression. The regression model is adjusted for age, sex and body mass index. The solid line shows hazard ratios and the shaded area shows $95 \%$ confidence intervals. Density plots show the distribution of calcium and white vertical lines indicate 2.5th, 25th, 50th, 75th and 97.5th percentiles.

CVD and cancer had minor impact on the association between high levels of calcium and PTH and VTE risk.

The observed association between high serum levels of PTH, calcium and VTE risk may be explained by a direct relationship. Calcium is an essential co-factor in the coagulation cascade, and several studies have shown associations between calcium, PTH and concentrations of hemostatic factors and coagulation activators (3, 4, 5), suggesting that high calcium and PTH causes an imbalance in the hemostatic system in an unfavorable prothrombotic direction. In a study of 35 patients with primary hyperparathyroidism and 25 control subjects, PAI-1 was found to be higher in the patients than that in the controls, and correlated with PTH levels in all subjects (3). In a case-control study, patients with primary hyperparathyroidism had higher levels of factor VII, factor $\mathrm{X}$ and D-dimer levels compared to controls (4), and a later case-control study revealed that patients with primary hyperparathyroidism had increased PAI-1 and lowered TFPI levels compared to those in controls (5). High levels of these factors have been associated with increased VTE risk in several studies $(17,38,39,40)$.

Despite a reasonable biological rationale for the impact of high serum calcium and PTH on VTE risk, we also observed an apparent higher VTE risk in subjects with low levels of calcium and PTH. The mechanism(s) underlying this surprising finding is not known. However, a recent meta-analysis reported an inverse association between calcium and subsequent breast cancer risk in prospective cohorts, and the results did not alter when they restricted the analyses to participants with calcium levels within the normal range (41). There is also several mechanisms by which cancer can cause low serum PTH in cancer subjects (42). However, the apparent association between low levels of calcium and PTH and VTE risk in our study remained unchanged after censoring for cancer.

The main strengths of our study include the prospective design, the large number of participants recruited from a general population, the high attendance rate, the longterm follow-up and the validated VTE events. As calcium and PTH are modifiable, some participants' individuals risk profiles may change during follow-up, leading to regression dilution bias and an underestimation of the associations. The longer the time is between exposure assessment and disease manifestation, the more present is this issue. However, an advantage of our study is the repeated measurements of participant characteristics during follow-up, which allowed for changes in calcium and PTH over time. Moreover, the cause-specific approach eliminated the potential effect of MI, stroke and cancer on the risk of VTE. Conversely, our study has some limitations.

Table 5 Incidence rates (IR) and hazard ratios (HR) of total venous thromboembolism (VTE) by categories of serum total calcium and parathyroid hormone (PTH) concentrations.

\begin{tabular}{|c|c|c|c|}
\hline PTH (pmol/L) & Calcium (mmol/L) & Person-years & Events \\
\hline \multirow{3}{*}{$0.1-2.1$} & $1.77-2.31$ & 5607 & 19 \\
\hline & $2.32-2.44$ & 13369 & 27 \\
\hline & $2.45-3.00$ & 5881 & 18 \\
\hline \multirow[t]{3}{*}{$2.2-3.9$} & $1.77-2.31$ & 13661 & 48 \\
\hline & $2.32-2.44$ & 27972 & 82 \\
\hline & $2.45-3.00$ & 8743 & 23 \\
\hline \multirow[t]{3}{*}{$4.0-26.8$} & $1.77-2.31$ & 9691 & 34 \\
\hline & $2.32-2.44$ & 11919 & 40 \\
\hline & $2.45-3.00$ & 3852 & 23 \\
\hline
\end{tabular}

\begin{tabular}{c}
\hline $\mathbf{I R}^{*}$ \\
\hline $3.39(2.16-5.31)$ \\
$2.02(1.39-2.94)$ \\
$3.06(1.93-4.86)$ \\
$3.51(2.65-4.66)$ \\
$2.93(2.36-3.64)$ \\
$2.63(1.75-3.96)$ \\
$3.51(2.51-4.91)$ \\
$3.36(2.46-4.58)$ \\
$5.97(3.97-8.98)$ \\
\hline
\end{tabular}

\begin{tabular}{l}
\hline $\mathbf{H R}^{\mathbf{+}}$ \\
\hline $1.51(0.91-2.49)$ \\
$0.87(0.56-1.34)$ \\
$1.23(0.74-2.05)$ \\
$1.27(0.89-1.82)$ \\
1.00 (ref.) \\
$0.92(0.58-1.46)$ \\
$1.12(0.75-1.67)$ \\
$1.01(0.69-1.48)$ \\
$1.75(1.10-2.78)$ \\
\hline
\end{tabular}

\begin{tabular}{l}
\hline $\mathbf{H R}^{\mp}$ \\
\hline $1.63(0.98-2.69)$ \\
$0.93(0.60-1.44)$ \\
$1.28(0.77-2.14)$ \\
$1.33(0.92-1.90)$ \\
1.00 (ref.) \\
$0.95(0.60-1.52)$ \\
$1.13(0.75-1.69)$ \\
$1.00(0.68-1.47)$ \\
$1.78(1.12-2.84)$ \\
\hline
\end{tabular}

*Incidence rate per 1000 person-years; ${ }^{\dagger}$ adjusted for age as time-scale and sex; ${ }^{\star}$ adjusted for age as time-scale, sex and body mass index. 
Measurements of ionized calcium and albumin were not available in our study, and therefore, we used total serum calcium. Total serum calcium will not only reflect the calcium physiology but also be a function of the serum albumin level. Information on use of glucocorticoids was not available in our study and could therefore not be taken into consideration. Furthermore, creatinine was only available for $44 \%$ of the eligible population. Finally, the serum samples from 1994 had been stored frozen for many years before analysis, and there appeared to have been some degradation of PTH during storage (43). However, a strong correlation between serum PTH values from 1994 and 2001 do indicate that the degradation was similar in all samples (43). If there existed an uneven degradation, this would most likely mask rather than introduce an association between PTH and VTE.

In our population-based cohort study, serum levels of calcium and PTH separately were not associated with future risk of VTE, neither by a continuous nor by a categorical approach. However, subjects with high levels of both calcium and PTH had increased risk of VTE compared to subjects with normal calcium and PTH.

\section{Supplementary data}

This is linked to the online version of the paper at http://dx.doi.org/10.1530/ EJE-16-1037.

\section{Declaration of interest}

The authors declare that there is no conflict of interest that could be perceived as prejudicing the impartiality of this study.

\section{Funding}

K G Jebsen TREC is supported by an independent grant from Stiftelsen Kristian Gerhard Jebsen.

\section{Author contribution statement}

G Lerstad carried out statistical analyses and drafted the manuscript together with J B Hansen and S K Brækkan, E Brodin, J Svartberg, R Jorde and $J$ Brox were involved in critical revision of the manuscript. $S \mathrm{~K}$ Brækkan, J Brox, R Jorde and J B Hansen were involved in study design and data collection. G Lerstad and S K Brækkan had full access to the data and take full responsibility for its integrity and the accuracy of data analysis.

\section{References}

1 Wermers RA, Khosla S, Atkinson EJ, Achenbach SJ, Oberg AL, Grant CS \& Melton LJ 3rd. Incidence of primary hyperparathyroidism in Rochester, Minnesota, 1993-2001: an update on the changing epidemiology of the disease. Journal of Bone and Mineral Research 2006 21 171-177. (doi:10.1359/JBMR.050910)

2 Bouillon R, Carmeliet G \& Boonen S. Ageing and calcium metabolism. Baillieres Journal of Endocrinology and Metabolism 199711 341-365. (doi:10.1016/S0950-351X(97)80332-1)
3 Chertok-Shacham E, Ishay A, Lavi I \& Luboshitzky R. Biomarkers of hypercoagulability and inflammation in primary hyperparathyroidism. Medical Science Monitor 200814 CR628-CR632.

4 Erem C, Kocak M, Hacihasanoglu A, Yilmaz M, Saglam F \& Ersoz HO. Blood coagulation, fibrinolysis and lipid profile in patients with primary hyperparathyroidism: increased plasma factor VII and X activities and D-Dimer levels. Experimental and Clinical Endocrinology and Diabetes 2008116 619-624. (doi:10.1055/s-2008-1065365)

5 Erem C, Kocak M, Nuhoglu I, Yilmaz M \& Ucuncu O. Increased plasminogen activator inhibitor-1, decreased tissue factor pathway inhibitor, and unchanged thrombin-activatable fibrinolysis inhibitor levels in patients with primary hyperparathyroidism. European Journal of Endocrinology 2009160 863-868. (doi:10.1530/EJE-09-0069)

6 Lind L, Skarfors E, Berglund L, Lithell H \& Ljunghall S. Serum calcium: a new, independent, prospective risk factor for myocardial infarction in middle-aged men followed for 18 years. Journal of Clinical Epidemiology $1997 \mathbf{5 0}$ 967-973. (doi:10.1016/S08954356(97)00104-2)

7 Hagstrom E, Hellman P, Larsson TE, Ingelsson E, Berglund L, Sundstrom J, Melhus H, Held C, Lind L, Michaelsson K et al. Plasma parathyroid hormone and the risk of cardiovascular mortality in the community. Circulation 2009119 2765-2771. (doi:10.1161/ CIRCULATIONAHA.108.808733)

8 Heit JA. Venous thromboembolism: disease burden, outcomes and risk factors. Journal of Thrombosis and Haemostasis 20053 1611-1617. (doi:10.1111/j.1538-7836.2005.01415.x)

9 Silverstein MD, Heit JA, Mohr DN, Petterson TM, O'Fallon WM \& Melton LJ 3rd. Trends in the incidence of deep vein thrombosis and pulmonary embolism: a 25-year population-based study. Archives of Internal Medicine 1998158 585-593. (doi:10.1001/archinte.158.6.585)

10 Glynn RJ \& Rosner B. Comparison of risk factors for the competing risks of coronary heart disease, stroke, and venous thromboembolism. American Journal of Epidemiology 2005 162 975-982. (doi:10.1093/aje/ kwi309)

11 Robetorye RS \& Rodgers GM. Update on selected inherited venous thrombotic disorders. American Journal of Hematology $20016 \mathbf{8}$ 256-268. (doi:10.1002/ajh.10002)

12 Rosendaal FR. Venous thrombosis: a multicausal disease. Lancet 1999 353 1167-1173. (doi:10.1016/S0140-6736(98)10266-0)

13 Cushman M, Tsai AW, White RH, Heckbert SR, Rosamond WD, Enright P \& Folsom AR. Deep vein thrombosis and pulmonary embolism in two cohorts: the longitudinal investigation of thromboembolism etiology. American Journal of Medicine 2004117 19-25. (doi:10.1016/j.amjmed.2004.01.018)

14 Prandoni P, Bilora F, Marchiori A, Bernardi E, Petrobelli F, Lensing AW, Prins $\mathrm{MH} \&$ Girolami A. An association between atherosclerosis and venous thrombosis. New England Journal of Medicine $2003 \mathbf{3 4 8}$ 1435-1441. (doi:10.1056/NEJMoa022157)

15 White RH. The epidemiology of venous thromboembolism. Circulation 2003 107 I4-I8. (doi:10.1161/01.cir.0000078468.11849.66)

16 Nossent AY, Eikenboom JC \& Bertina RM. Plasma coagulation factor levels in venous thrombosis. Seminars in Hematology 200744 77-84. (doi:10.1053/j.seminhematol.2007.01.006)

17 Tsai AW, Cushman M, Rosamond WD, Heckbert SR, Tracy RP, Aleksic N \& Folsom AR. Coagulation factors, inflammation markers, and venous thromboembolism: the longitudinal investigation of thromboembolism etiology (LITE). American Journal of Medicine 2002 113 636-642. (doi:10.1016/S0002-9343(02)01345-1)

18 Jacobsen BK, Eggen AE, Mathiesen EB, Wilsgaard T \& Njolstad I. Cohort profile: the Tromso study. International Journal of Epidemiology 201241 961-967. (doi:10.1093/ije/dyr049)

19 Borch KH, Braekkan SK, Mathiesen EB, Njolstad I, Wilsgaard T, Stormer J \& Hansen JB. Abdominal obesity is essential for the risk of venous thromboembolism in the metabolic syndrome: the Tromso study. Journal of Thrombosis and Haemostasis 20097 739-745. (doi:10.1111/j.1538-7836.2008.03234.x) 
20 Kronborg J, Solbu M, Njolstad I, Toft I, Eriksen BO \& Jenssen T. Predictors of change in estimated GFR: a population-based 7-year follow-up from the Tromso study. Nephrology Dialysis Transplantation 200823 2818-2826. (doi:10.1093/ndt/gfn148)

21 Braekkan SK, Mathiesen EB, Njolstad I, Wilsgaard T, Stormer J $\&$ Hansen JB. Mean platelet volume is a risk factor for venous thromboembolism: the Tromso study, Tromso, Norway. Journal of Thrombosis and Haemostasis 20108 157-162. (doi:10.1111/j.15387836.2009.03498.x)

22 Rinde LB, Smabrekke B, Mathiesen EB, Lochen ML, Njolstad I, Hald EM, Wilsgaard T, Braekkan SK \& Hansen JB. Ischemic stroke and risk of venous thromboembolism in the general population: the Tromso study. Journal of the American Heart Association 20165. (doi:10.1161/JAHA.116.004311)

23 Rinde LB, Lind C, Smabrekke B, Njolstad I, Mathiesen EB, Wilsgaard T, Lochen ML, Hald EM, Vik A, Braekkan SK et al. Impact of incident myocardial infarction on the risk of venous thromboembolism: the Tromso study. Journal of Thrombosis and Haemostasis $2016 \mathbf{1 4}$ 1183-1191. (doi:10.1111/jth.13329)

24 Blix K, Braekkan SK, le Cessie S, Skjeldestad FE, Cannegieter SC $\&$ Hansen JB. The increased risk of venous thromboembolism by advancing age cannot be attributed to the higher incidence of cancer in the elderly: the Tromso study. European Journal of Epidemiology 2014 29 277-284. (doi:10.1007/s10654-014-9902-7)

25 Blondon M, Rodabough RJ, Budrys N, Johnson KC, Berger JS, Shikany JM, Raiesdana A, Heckbert SR, Manson JE, LaCroix AZ et al. The effect of calcium plus vitamin D supplementation on the risk of venous thromboembolism. From the Women's Health Initiative Randomized Controlled Trial. Journal of Thrombosis and Haemostasis 2015113 999-1009. (doi:10.1160/TH14-05-0478)

26 van Ballegooijen AJ, Reinders I, Visser M \& Brouwer IA. Parathyroid hormone and cardiovascular disease events: a systematic review and meta-analysis of prospective studies. American Heart Journal 2013165 655-664, 664.e651-e655. (doi:10.1016/j. ahj.2013.02.014)

27 Reid IR, Gamble GD \& Bolland MJ. Circulating calcium concentrations, vascular disease and mortality: a systematic review. Journal of Internal Medicine 2016279 524-540. (doi:10.1111/joim.12464)

28 Jorde R, Bonaa KH \& Sundsfjord J. Population based study on serum ionised calcium, serum parathyroid hormone, and blood pressure. The Tromso study. European Journal of Endocrinology 1999141 350-357. (doi:10.1530/eje.0.1410350)

29 Jorde R, Sundsfjord J, Fitzgerald P \& Bonaa KH. Serum calcium and cardiovascular risk factors and diseases: the Tromso study. Hypertension 199934 484-490. (doi:10.1161/01.HYP.34.3.484)

30 Braekkan SK, Mathiesen EB, Njolstad I, Wilsgaard T, Stormer J \& Hansen JB. Family history of myocardial infarction is an independent risk factor for venous thromboembolism: the Tromso study. Journal of Thrombosis and Haemostasis 20086 1851-1857. (doi:10.1111/j.15387836.2008.03102.x)

31 Lind C, Enga KF, Mathiesen EB, Njolstad I, Braekkan SK \& Hansen JB. Family history of myocardial infarction and cause-specific risk of myocardial infarction and venous thromboembolism: the
Tromso study. Circulation Cardiovascular Genetics 20147 684-691. (doi:10.1161/CIRCGENETICS.114.000621)

32 Quist-Paulsen P, Naess IA, Cannegieter SC, Romundstad PR, Christiansen SC, Rosendaal FR \& Hammerstrom J. Arterial cardiovascular risk factors and venous thrombosis: results from a population-based, prospective study (the HUNT 2). Haematologica 201095 119-125. (doi:10.3324/haematol.2009.011866)

33 Braekkan SK, Hald EM, Mathiesen EB, Njolstad I, Wilsgaard T, Rosendaal FR \& Hansen JB. Competing risk of atherosclerotic risk factors for arterial and venous thrombosis in a general population: the Tromso study. Arteriosclerosis, Thrombosis, and Vascular Biology 201232 487-491. (doi:10.1161/ATVBAHA.111.237545)

34 Hagstrom E, Lundgren E, Lithell H, Berglund L, Ljunghall S, Hellman P \& Rastad J. Normalized dyslipidaemia after parathyroidectomy in mild primary hyperparathyroidism: population-based study over five years. Clinical Endocrinology 200256 253-260. (doi:10.1046/j.0300-0664.2001.01468.x)

35 Sorensen HT, Horvath-Puho E, Sogaard KK, Christensen S, Johnsen SP, Thomsen RW, Prandoni P \& Baron JA. Arterial cardiovascular events, statins, low-dose aspirin and subsequent risk of venous thromboembolism: a population-based case-control study. Journal of Thrombosis and Haemostasis 20097 521-528. (doi:10.1111/j.15387836.2009.03279.x)

36 Sorensen HT, Horvath-Puho E, Lash TL, Christiansen CF, Pesavento R, Pedersen L, Baron JA \& Prandoni P. Heart disease may be a risk factor for pulmonary embolism without peripheral deep venous thrombosis. Circulation 2011124 1435-1441. (doi:10.1161/ CIRCULATIONAHA.111.025627)

37 Stewart AF. Clinical practice. Hypercalcemia associated with cancer. New England Journal of Medicine 2005352 373-379. (doi:10.1056/ NEJMcp042806)

38 Andreescu AC, Cushman M \& Rosendaal FR. D-dimer as a risk factor for deep vein thrombosis: the Leiden Thrombophilia study. Journal of Thrombosis and Haemostasis 200287 47-51.

39 Meltzer ME, Lisman T, de Groot PG, Meijers JC, le Cessie S, Doggen CJ $\&$ Rosendaal FR. Venous thrombosis risk associated with plasma hypofibrinolysis is explained by elevated plasma levels of TAFI and PAI-1. Blood 2010116 113-121. (doi:10.1182/blood-2010-02-267740)

40 Dahm A, Van Hylckama Vlieg A, Bendz B, Rosendaal F, Bertina RM $\&$ Sandset PM. Low levels of tissue factor pathway inhibitor (TFPI) increase the risk of venous thrombosis. Blood 2003101 4387-4392. (doi:10.1182/blood-2002-10-3188)

41 Wulaningsih W, Sagoo HK, Hamza M, Melvin J, Holmberg L, Garmo H, Malmstrom H, Lambe M, Hammar N, Walldius G et al. Serum calcium and the risk of breast cancer: findings from the Swedish AMORIS study and a meta-analysis of prospective studies. International Journal of Molecular Sciences 2016 17. (doi:10.3390/ijms17091487)

42 Schattner A, Dubin I, Huber R \& Gelber M. Hypocalcaemia of malignancy. Netherlands Journal of Medicine $2016 \mathbf{7 4} 231-239$.

43 Jorde R, Svartberg J \& Sundsfjord J. Serum parathyroid hormone as a predictor of increase in systolic blood pressure in men. Journal of Hypertension 200523 1639-1644. (doi:10.1097/01. hjh.0000179764.40701.36)

Received 17 December 2016

Revised version received 21 February 2017

Accepted 27 February 2017 